\title{
Welche Faktoren bestimmen das Rupturrisiko?
}

Fragestellung: Wie sieht die „natural history“ von nicht rupturierten Aneurysmen aus?

Hintergrund: Besonders durch die breite Anwendung von MR und MR-Angiografie werden intrakranielle Aneurysmen als Zufallsbefunde gefunden. Das Rupturrisiko dieser Aneurysmen ist individuell schwer einzuschätzen. Die vorliegende Studie untersucht an einem großen Kollektiv, welche Risikofaktoren die Ruptur eines Aneurysmas begünstigen.

Patienten und Methodik: Von Januar 2001 bis April 2004 wurden 5.720 Patienten mit Aneurysmen in die Studie eingeschlossen. Alle Aneurysmen hatten eine Größe von mindestens $3 \mathrm{~mm}$.

Ergebnisse: Insgesamt wurden 6.697 Aneurysmen in der Studie gesehen. Davon wurden $91 \%$ inzidentell gefunden. Die meisten Aneurysmen waren an der A. cerebri media (36\%) und der A. carotis interna (34\%) lokalisiert. Die mittlere Größe der Aneurysmen betrug 5,7 mm. Während der Follow-up-Periode, die insgesamt 11.660 Aneurysma-Jahre betrug, wurden Rupturen bei 111 Patienten gesehen. Dies entspricht einer jährlichen Rupturrate von $0,95 \%$. Das Rupturrisiko stieg deutlich mit größerem Aneurysma-Durchmesser an ( $\downarrow$ Abb. 1). Nahm man die An-

UCAS Japan Investigators. The natural course of unruptured cerebral aneurysms in a Japanese cohort. N Engl J Med 2012; 366: 2474-82 eurysmen zwischen $3 \mathrm{~mm}$ und $4 \mathrm{~mm}$ als Referenz, betrug das relative Rupturrisiko bei Aneurysmen zwischen $5 \mathrm{~mm}$ und $6 \mathrm{~mm} \mathrm{1,13,} \mathrm{zwi-}$

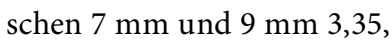
zwischen $10 \mathrm{~mm}$ und $24 \mathrm{~mm}$

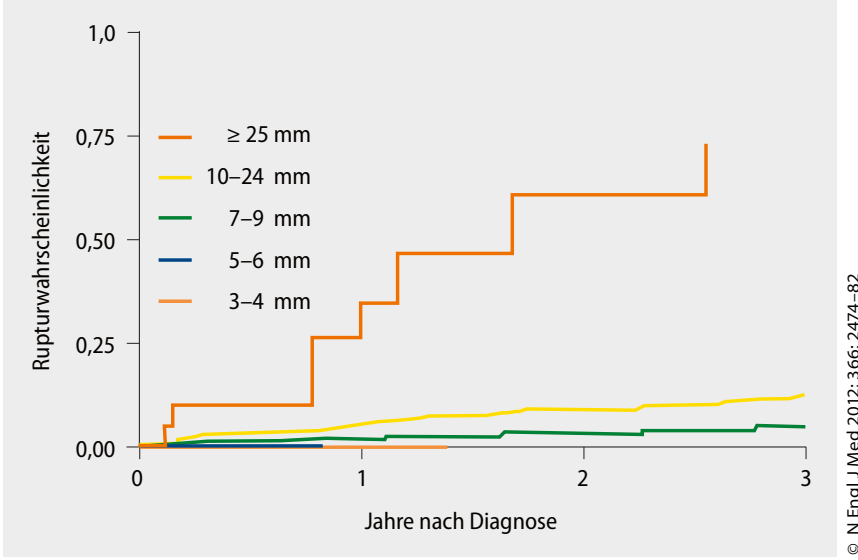

1 Die Rupturwahrscheinlichkeit stieg mit der Aneurysmagröße.

9,09 und bei Aneurysmen größer als $25 \mathrm{~mm}$ 76,26. Vergleicht man die Aneurysmen der A. cerebri media mit denen in der hinteren Zirkulation und mit denen des Ramus communicans anterior, betrug das Rupturrisiko bei Letzteren relativ 1,90. Ebenfalls Einfluss auf das Rupturrisiko hatte die Aneurysma-Konfiguration (insbesondere Tochter-Aneurysmen). Hier betrug dann das relative Rupturrisiko 1,63.

Schlussfolgerung: Die japanischen Autoren schlussfolgern, dass das Rupturrisiko bisher nicht rupturierter Aneurysmen von der Größe des Aneurysmas, der Lokalisation des Aneurysmas und der Form des Aneurysmas abhängig ist.

\section{- Kommentar von Prof. Michael Forsting}

\section{Ein weiterer Mosaikstein auf dem Weg zur optimierten Beratung}

Diese aus Japan vorgelegte Studie bestätigt auf der einen Seite die schon bekannten Fakten, dass die Aneurysma-Größe ein wichtiger Risikofaktor ist. Auf der anderen Seite ergänzen die Japaner jetzt, dass auch die Form des Aneurysmas eine Rolle spielt. Dies ist unter "Aneurysma-Therapeuten" (egal ob neurochirurgisch oder neuroradiologisch) schon lange anerkannt, ist jedoch in der neurologischen Community bislang immer auf Zweifel gestoßen. Die japanischen Autoren räumen zusätzlich ein, dass ihre Studie natürlich einem Bias unterliegt. Insgesamt wurden 2.000 Aneurysmen operiert und nicht nachbeobachtet und die Autoren konzidieren, dass diese Aneurysmen wahrscheinlich ebenfalls eine intuitive Risikokonstellation in sich bargen (z. B. Asymmetrie von A1-Segmenten am Ramuscommunicans-anterior-Aneurysmen), sodass das wahre Rupturrisiko wahrscheinlich höher gewesen wäre, wenn man alle
Patienten nur beobachtet hätte. Insgesamt ist diese Studie ein weiterer Mosaikstein der uns hilft, Patienten mit nicht rupturierten Aneurysmen immer besser zu beraten.

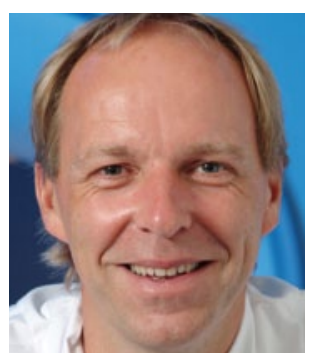

Prof. Dr. med. Michael Forsting, Essen

Direktor des Instituts für Diagnostische und Interventionelle Radiologie und Neuroradiologie, Uni-Klinikum Essen E-Mail: michael.forsting@uk-essen.de 